\title{
Solar prominence polarimetry
}

\author{
E. Wiehr ${ }^{1}$ and M. Bianda ${ }^{2}$ \\ 1 Universitäts-Sternwarte, Geismarlandstraße 11, 37083 Göttingen, Germany \\ 2 Istituto Ricerche Solari, Via Patocchi, 6644 Locarno-Orselina, Switzerland
}

Received 7 April 2003 / Accepted 6 May 2003

\begin{abstract}
We measure the resonance polarization in solar prominences in $\mathrm{H} \alpha, \mathrm{H} \beta$ and $\mathrm{HeD}_{3}$. A two-dimensional set-up with narrow-band filter, polarization analyzer and CCD camera is used to take prominence images in polarized light at high spatial resolution. Placed on a coudé telescope's hour axis, the observations near the equinoxia are free from purely instrumental polarization. Above the $0.1 \%$ noise limit, the Balmer lines do not show a polarization in contrast to the $\mathrm{HeD}_{3}$ line. Here, we determine the complete polarization profile after exchange of filter and CCD with the spectrograph, keeping the polarization analyzer fixed. In most prominences the Stokes- $U$ and $-Q$ profiles are not similar to Stokes- $I$ : occasionally the blue and the red components of the emission are equal or even show a reverse ratio. This fits calculations for magnetic field strengths of the order of 50 Gauß being markedly stronger than commonly assumed.
\end{abstract}

Key words. Sun: atmosphere - Sun: prominences - instrumentation: polarimeters - techniques: polarimetry

\section{Introduction}

Solar prominences are predominantly illuminated from below. This asymmetric incidence of light yields a partial polarization of that part of the emission which is formed by scattering processes. The already small amount of resonance polarization is further diminished by the Hanle effect if the emitting plasma is embedded in a magnetic field (Sahal-Bréchot et al. 1977). The resulting small amount of polarized light is superposed by the usually much larger polarization of the instrument. Extended measurements of linear polarization in prominences were made by Leroy et al. (1984) through filters integrating the whole emission line profiles. They found values up to several percent in $\mathrm{H} \alpha, \mathrm{H} \beta$, and $\mathrm{He}_{3}$.

Landi degl'Innocenti (1982) shows that the distribution of polarization through the $\mathrm{He}_{3}$ fine-structure components offers a possibility to determine more than the two magnetic field parameters deduced from integrated data of the whole emission line by Leroy et al. (1984). This, however, requires full spectral resolution of polarization profile of $\mathrm{He}_{3}$. Athay et al. (1983) find that the spectral distribution of the intensity differs from that of the linear polarization through the He triplet. They argue that this effect can hardly be due to optical thickness effects and might be due to different sensibility of the He fine-structure components to the Hanle effect. Since the determination of the prominence magnetic fields essentially depends on such measurements, it is worth-while to verify the prominence polarization with different methods.

Send offprint requests to: $\mathrm{E}$. Wiehr, e-mail: ewiehr@uni-sw.gwdg.de

\section{Observing method}

A telescope with a "German type" coudé mounting (as, e.g., the Gregory telescopes at Locarno and at Tenerife; Wiehr 1987), is most suitable for polarimetry. Its instrumental polarization originates almost entirely from the two folding flat mirrors, the relative orientation of which varying only with the solar declination. Their combined influence is thus small and largely constant over a day (cf. Wiehr 1974); it even vanishes for zero solar declination at the equinoxia, where the two deflections are precisely orthogonal. We observed close to several equinoxia with the Gregory Coudé Telescope at Tenerife until its dismantling in spring 2002, and in autumn 2002 with its "twin" at Locarno.

The polarized light was measured with a $\lambda / 2$ plate followed by a calcite (Savart plate), a narrow-band filter, and a CCD camera placed directly on the telescope's hour axis (Wiehr \& Bianda 2002). The filters of a few $\AA$ widths were accurately centered on $\mathrm{H} \alpha, \mathrm{H} \beta$, and $\mathrm{HeD}_{3}$, respectively, by electronically controlled heating and suitable tilt, controlled with the spectrograph. The finite field-of-view required for the Savart plate, was defined by a $40^{\prime \prime} \times 120^{\prime \prime}$ aperture in front of the polarization optics which was imaged by telecentric optics on the CCD. Lenses, filter, and CCD could readily be removed for an alternate use of the spectrograph, keeping the polarization analyzer unchanged. This allowed intermediate spectroscopic measurements of the whole polarization line profile for such prominences which show a signal in the 2-D polarimetric setup.

The linear polarization was measured with the "beamexchange technique" (Semel et al. 1993) where two exposures 


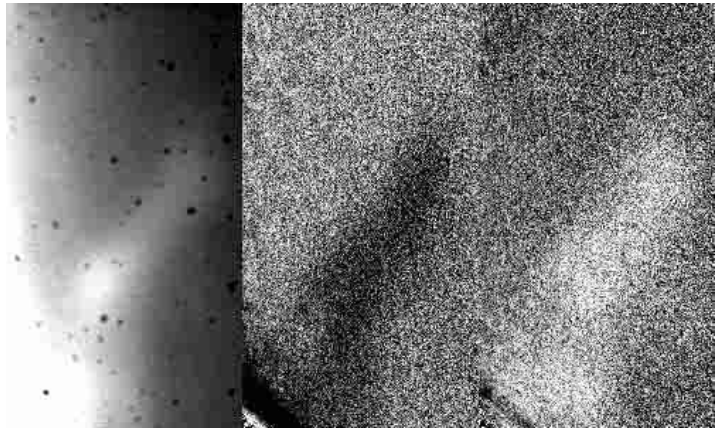

Fig. 1. Prominence observed on Sep. 26, 2002, at the east limb, $22^{\circ} \mathrm{N}$ in the $\mathrm{He}_{3}$ emission through a $5 \AA$ filter (left panel) together with images in the light of Stokes- $Q$ (middle) and $U$ (right) showing maxima near $1 \%$; image size $40^{\prime \prime} \times 70^{\prime \prime}$.

are taken sucessively with $0^{\circ}$ and $45^{\circ}$ orientation of the half wave plate in front of the Savart plate. The circular polarization was measured replacing the $\lambda / 2$ by a $\lambda / 4$ plate. Using the algorithm described by Bianda et al. (1998), uncertainties of the CCD's gain table do not affect the measurements. The "beam-exchange technique" requires largely equal location and sharpness of the solar features in the two exposures. The first condition is assured by the high accuracy of the primary image guider (Küveler et al. 1998) which compensates drifts in the telescope pointing with high accuracy. Remaining differences in the features' precise locations on the CCD, as well as different image sharpness, were minimized by frame selection among several exposures, yielding a highly similar pair of subimages. The fixed orientation of the Savart plate affects that the $Q+$ direction is parallel to the CCD rows and not to the solar limb.

Although the instrumental polarization is expected to be very small near zero solar declination (see above), we measured it at disk center with reasonable diminution of the light level by neutral filters. The obtained value of typically $5 \times 10^{-4}$ shows that near the equinox a coudé type telescope with "German mounting" is, indeed, largely free from linear polarization, and our final data is unaffected by the telescope. The small disk center value is nevertheless subtracted from the prominence observations, assuming that it does not vary over the disk (the maximum declination difference being only $0.25^{\circ}$ ).

Our 2-D polarimeter has been proven to yield high spatial and polarimetric accuracy (Wiehr \& Bianda 2003). The integration over small wavelength ranges of the filters assures a complete covering of the respective emission line, independent on Doppler shifts. It furthermore yields a good spatial resolution due to the short exposure times of typically $50 \mathrm{~ms}$, and thus allows a daily check of all prominences within a short time interval (Wiehr 2002). The resulting polarization images can then be used for a selection of prominences worse being further observed with the spectroscopic polarimeter setup.

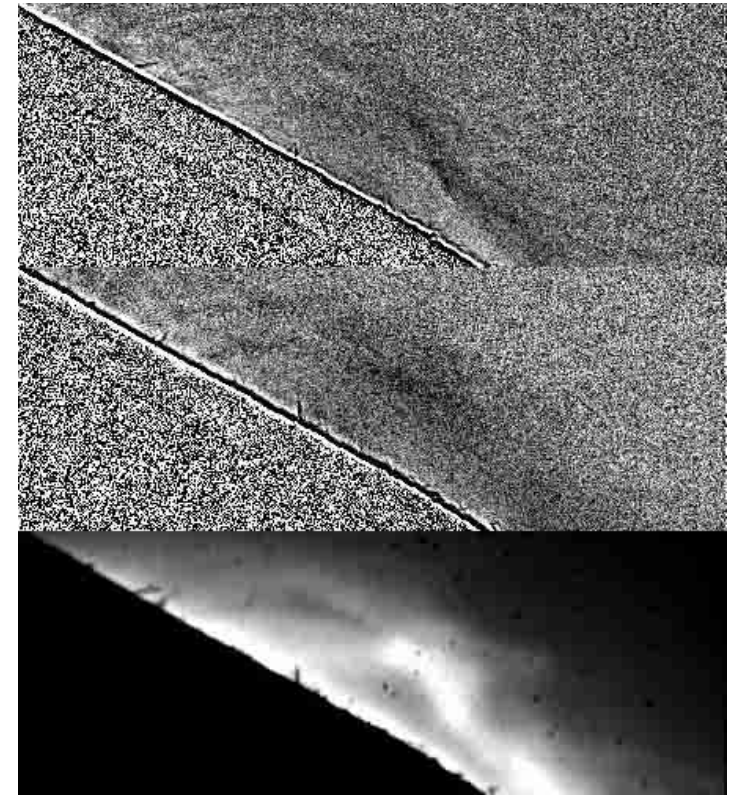

Fig. 2. Prominence observed on Sep. 20, 2002, at the west limb, $10^{\circ} \mathrm{S}$ in the $\mathrm{He} \mathrm{D}_{3}$ emission through a $5 \AA$ filter (lower panel; solar disk masked) together with images in the light of Stokes- $Q$ (upper) and $U$ (middle); image size $120^{\prime \prime} \times 40^{\prime \prime}$.

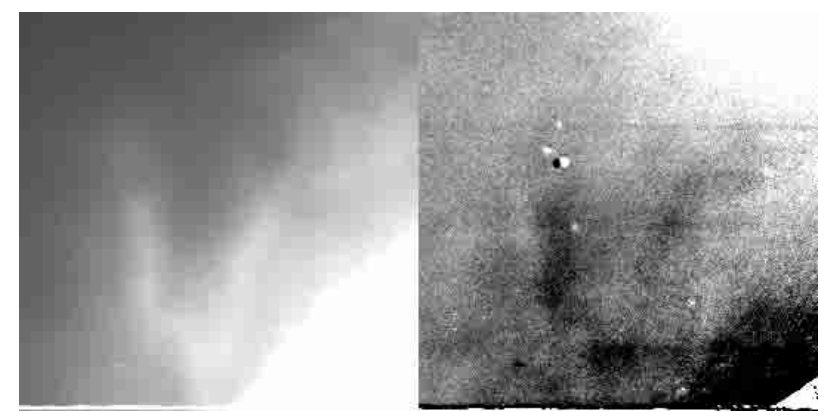

Fig. 3. Prominence observed on March 24, 2002, at the east limb, $45^{\circ} \mathrm{S}$ in the $\mathrm{He}_{3}$ emission through a $5 \AA$ filter (left panel) and in the light of Stokes- $U / I$ (i.e. parallel to the limb, $Q$ being defined parallel to the $\mathrm{CCD}$ rows); image size $80^{\prime \prime} \times 80^{\prime \prime}$

\section{Results}

\subsection{Polarization images of prominences}

During the various observing campaigns at four equinoxia, we observed dozens of prominences occurring above the limb at various solar latitudes and with large variety of brightness (i.e. optical thickness; cf. Stellmacher \& Wiehr 1995). A measurable linear polarization was not found in the $\mathrm{H \alpha}$ or the $H \beta$ lines. Examples of $\mathrm{He}_{3}$ linear polarization images are shown in Figs. 1, 2, and 3. The spatial variation of the linear polarization differs from that of the intensity features. This is most pronounced for the prominence in Fig. 3, where the two branches behave oppositely in $U / I$ as in intensity. 


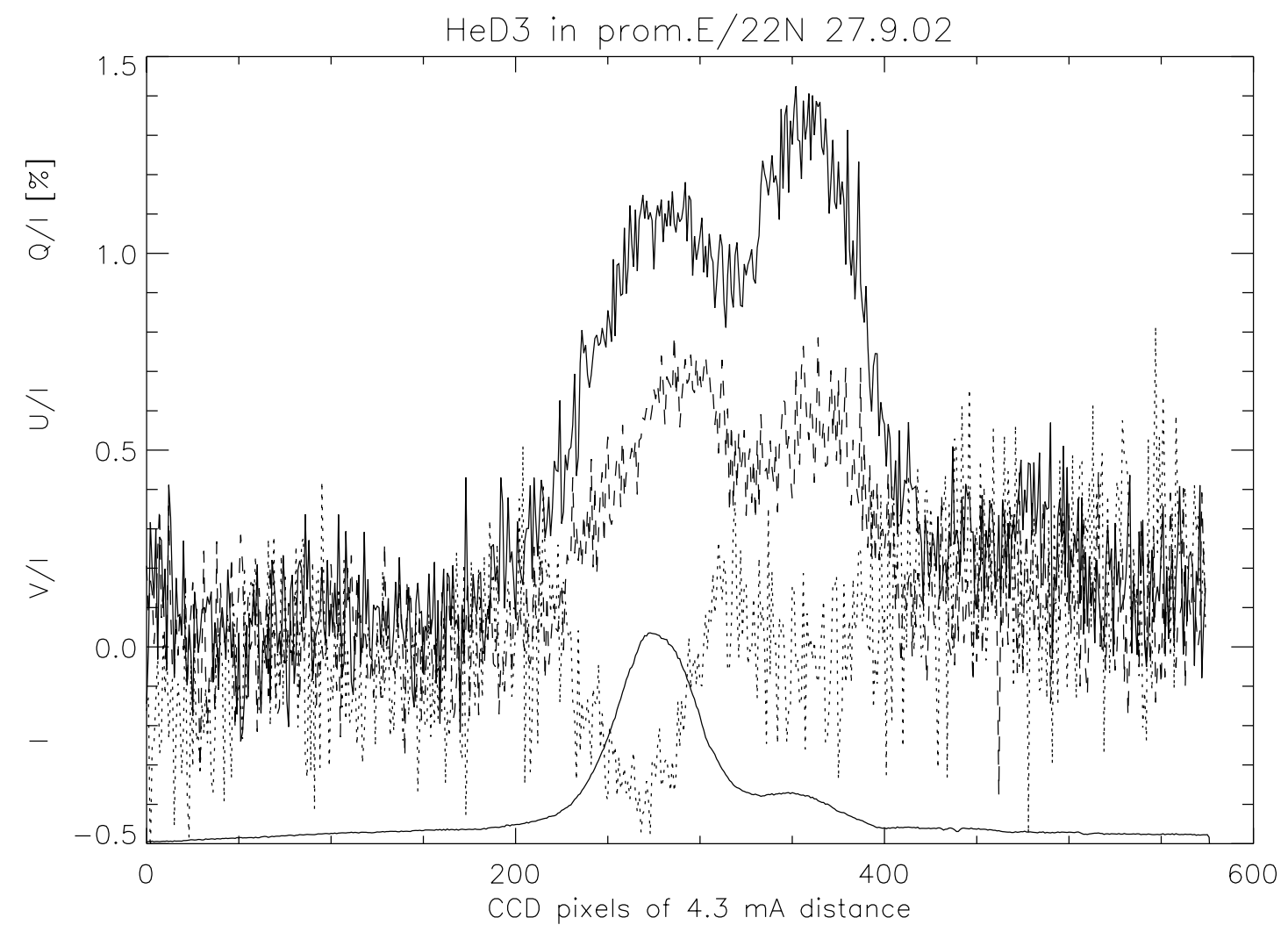

Fig. 4. Spectral scan of the $\mathrm{He} \mathrm{D}_{3}$ polarization in a prominence at the east limb, $18^{\circ} \mathrm{N}$, observed on Sep. 27,2002 ; full line $=Q / I$, dashes $=$ $U / I$, dots $=V / I$, together with the intensity profile (thin full line) showing the composed red and the well separated faint blue components.

\subsection{Polarization through the $\mathrm{He}_{3}$ emission profile}

For a more detailed investigation of such a behavior, the spectral distribution of the linear polarization through the whole $\mathrm{HeD}_{3}$ profile was measured with the spectrograph mode of our polarimeter. Five of the six $\mathrm{HeD}_{3}$ components superpose to an (unresolved) blue emission, whereas the sixth faint component at $343.3 \mathrm{~m} \AA$ red-wards remains well separated (cf. Landi degl'Innocenti 1982). We find that the intensity ratio of the two observable components is mostly near $6: 1$, being close to the ranges found by Athay et al. (1983) and by Lopez-Ariste \& Casini (2002). This significant deviation from the 8:1 ratio, expected from the transition probabilities, is a strong hint for non-negligible optical thickness. Similarly, Stellmacher et al. (2003) calculate for the He 10830 triplet that the 8:1 ratio of its combined two red components and its separate faint blue component declines to $6: 1$ already for $\tau \approx 0.4$. The $\mathrm{HeD}_{3}$ lines will not behave much differently.

Among those prominences which yield polarization above $10^{-3}$, the majority shows a ratio of the (combined) blue component(s) and the faint red component which is smaller than the ratio of the Stokes- $I$. This result agrees with findings by Paletou et al. (2001) and by Lopez-Ariste \& Casini (2002). For $\mathrm{HeD}_{3}$ profiles which show $P_{\text {lin }}^{\text {red }}>1.0 \%$, we find a significantly smaller difference between the two $\mathrm{HeD}_{3}$ emissions: in Fig. 4 the $Q / I$ and the $U / I$ maxima of the (single) red component exceed those of the (stronger) blue component(s). In these rare cases, we also measure a Stokes- $V$ signal with typically $V / I<0.5 \%$ (cf. Fig. 4).

\section{Conclusion}

Disregarding the fact that the calculations of fractional linear polarization by Landi degl'Innocenti (1982) and by Lopez Ariste \& Casini (2002) are not rigorously valid for nonnegligible optical thickness, a nearly equal polarization in the the two components indicates field strengths up to $50 \mathrm{Gauß}$. Such strong fields were already measured via Zeeman effect by Kim et al. (1984) and later by Paletou et al. (2001). Also LopezAriste \& Casini (2002) deduce from the re-considered data by Athay et al. (1983) similar field strengths which significantly exceed the values found by Leroy et al. (1984, and references therein). Such higher field strengths might agree with higher gas-pressures deduced from the observed ratio of the $\mathrm{Ca}^{+} 8452$ and $\mathrm{H} \beta$ emissions by Stellmacher \& Wiehr (2000; and references therein).

The absence of linear polarization in the $\mathrm{H} \alpha$ and $\mathrm{H} \beta$ emissions seems to disagree with Leroy et al. (1984) who found values of several percent, which should easily have been detected at our 2-D polarimetric accuracy of $10^{-3}$. Leroy (1981) finds that the linear polarization decreases with brightness of the Balmer emissions. This decrease, however, only occurs if the optical thickness in the $\mathrm{H} \alpha$ line exceeds unity optical thickness, which corresponds to an integrated line emission above $5 \times 10^{4} \mathrm{erg} /\left(\mathrm{cm}^{2}\right.$ s ster) (cf. Stellmacher \& Wiehr 1994; Fig. 4). We may reasonably exclude a preferred selection of brightest prominences for our observations; the large quantity of prominences observed near four equinoxia with our 2-D polarimeter, 
certainly contains much fainter ones - at least such with less bright locations. It it thus astonishing that none of the 2-D polarization images indicates any significant signal of the Balmer lines in excess of $10^{-3}$. (A similar finding is reported by the Zürich group.)

As far as the $\mathrm{H} \beta$ line is concerned, its decrease of linear polarization with brightness can hardly be explained by optical thickness since that emission is 5-11 times fainter than $\mathrm{H} \alpha$ (Stellmacher \& Wiehr 1994; Fig. 3). Bommier et al. (1986) discuss the influence of electron density; according to their Fig. 3, our upper limit of the $10^{-3}$ level would indicate an electron density of almost $10^{11} \mathrm{~cm}^{-3}$ which seems to be rather high - even with regard to the higher gas-pressure favored by Stellmacher \& Wiehr (2000) and with the higher prominence magnetic fields indicated by the polarimetry. It remains unclear why the majority of prominences does not yield linear polarization in the Balmer lines above the $10^{-3}$ level. Stronger magnetic fields might decrease the resonance polarization, but would increase the circular polarization; this should be the subject of future observations.

Acknowledgements. We are indebted to Drs. G. Stellmacher and E. Landi degl'Innocenti for fruitful discussions and suggestions. The Gregory Coudé telescope at Locarno is operated by the "Istituto Ricerche Solari" at the Swiss observatory of Orselina near Locarno. The Gregory Coudé telescope on Tenerife is operated by the Universitäts-Sternwarte, Göttingen (USG), at the Spanish "Observatorio del Teide" of the Instituto de Astrofísica de Canarias.

\section{References}

Athay, R. G., Querfeld, C. W., Smartt, R. N., Landi degl'Innocenti, E., \& Bommier, V., 1983, Sol. Phys., 89, 3

Bianda, M., Solanki, S., \& Stenflo, J. O. 1998, A\&A, 331, 760

Bommier, V., Leroy, J. L., \& Sahal-Bréchot, S. 1986, A\&A, 156, 90

Kim, I. S., Koutchmy, S., Stellmacher, G., \& Nikolskii, G. M. 1984, A\&A, 140, 112

Küveler, G., Wiehr, E., Thomas, M., et al. 1998, Sol. Phys., 182, 247

Landi degl'Innocenti, E. 1982, Sol. Phys., 79, 291

Leroy, J. L. 1981, Sol. Phys., 71, 285

Leroy, J. L., Bommier, V., \& Sahal-Bréchot, S. 1984, A\&A, 131, 33

Lopez-Ariste, A., \& Casini, R. 2002, ApJ, 575, 529

Paletou, F., Lopez Ariste, A., Bommier, V., \& Semel, M. 2001, A\&A, 375, L41

Sahal-Bréchot, S., Bommier, V., \& Leroy, J. L. 1977, A\&A, 59, 223

Semel, M., Donati, J.-F., \& Rees, D. E. 1993, A\&A, 278, 231

Stellmacher, G., \& Wiehr, E. 1994, A\&A, 290, 655

Stellmacher, G., \& Wiehr, E. 1995, A\&A, 299, 921

Stellmacher, G., \& Wiehr, E. 2000, Sol. Phys., 196, 357

Stellmacher, G., Wiehr E., \& Dammasch, I. 2003, Sol. Phys., submitted

Wiehr, E. 1974, Sol. Phys., 35, 343

Wiehr, E. 1987, in The Role of Fine-Scale Magnetic Fields on the Structure of the Solar Atmosphere, ed. E. H. Schröter, M. Vázquez, \& A. A. Wyller (Cambridge Univ. Press), 354

Wiehr, E. 2002a, in Magnetic Coupling of the Solar Atmosphere, ed. H. Sawaya-Lacoste (ESA Pub), SP-505, in press

Wiehr, E., \& Bianda, M. 2003, A\&A, 398, 739 